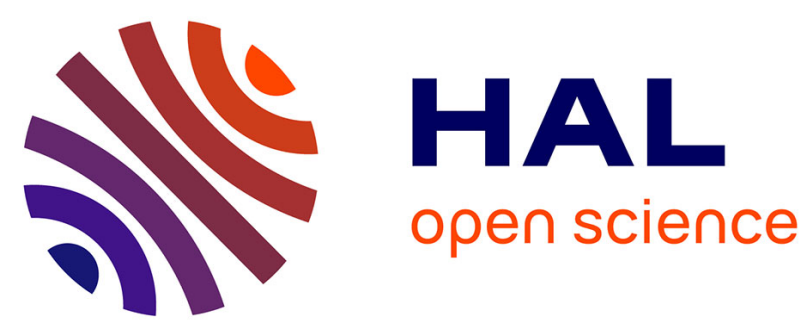

\title{
Extraction of EI and EDM muscle sources from surface electromyographic signals using delay estimation
}

\author{
Anton Dogadov, Christine Serviere, Franck Quaine
}

\section{To cite this version:}

Anton Dogadov, Christine Serviere, Franck Quaine. Extraction of EI and EDM muscle sources from surface electromyographic signals using delay estimation. BioSMART 2016 - International Conference on Bio-engineering for Smart Technologies, Dec 2016, Dubaï, United Arab Emirates. 10.1109/BIOSMART.2016.7835471 . hal-01418648

\section{HAL Id: hal-01418648 \\ https://hal.science/hal-01418648}

Submitted on 22 Jan 2017

HAL is a multi-disciplinary open access archive for the deposit and dissemination of scientific research documents, whether they are published or not. The documents may come from teaching and research institutions in France or abroad, or from public or private research centers.
L'archive ouverte pluridisciplinaire HAL, est destinée au dépôt et à la diffusion de documents scientifiques de niveau recherche, publiés ou non, émanant des établissements d'enseignement et de recherche français ou étrangers, des laboratoires publics ou privés. 


\title{
Extraction of EI and EDM muscle sources from surface electromyographic signals using delay estimation
}

\author{
Anton Dogadov, Christine Serviere, Franck Quaine \\ Univ. Grenoble Alpes, GIPSA-Lab, \\ CNRS, GIPSA-Lab \\ Grenoble, France \\ \{anton.dogadov, christine.serviere, franck.quaine\}@gipsa-lab.grenoble-inp.fr
}

\begin{abstract}
Interference or a crosstalk from nearby muscles is a classic problem in surface electromyographic recordings. Most studies of crosstalk diminution have only focused on blind source separation techniques and spatial filtering of simulated signals. The aim of this study was to perform an extraction of the activity of index and little finger extensor muscles from the electromyographic mixtures recorded with a surface electrode array. The motor unit action potential were detected in the mixtures and classified according to an inter-electrode delay. The signal-to-interference ratio performance of the proposed method was higher than performance of the beamformer and the frequency JADE algorithms. The findings show that creation of a muscle action potential propagation model, required by beamformer, could be problematic. The proposed algorithm uses a generalized model and shows higher performance.
\end{abstract}

Keywords-surface electromyogram, interference reduction, signal extraction, delay estimation

\section{INTRODUCTION}

The muscles are spatially extended signal sources. They consist of fibers, associated in motor units (MU), which are the smallest functional units of muscles. A muscle is activated by a neural output that is transmitted by motor nerves, which enter the muscle belly in the motor points. There are usually multiple (up to 5) motor points in finger extensor muscles, situated close to each other [1]. When a muscle is activated, the motor unit action potential (MUAP, depolarization) is propagated in each MU along the muscle body from the motor point to both ends of the muscle. The mean speed of MUAP propagation is around $4 \mathrm{~m} / \mathrm{s}$ [2].

During surface electromyographic (sEMG) measurements, surface electrodes, situated on the skin, record the muscle activity that is spatially filtered by a volume conductor (muscles, fat and skin).Moreover, as some muscles are situated close to each other, a surface electrode usually records activity of a muscle of interest perturbed by signals from other muscles, active at the same time. This disturbance is called a crosstalk or an interference. The interference from nearby muscles is a common problem for sEMG applications as prosthesis control or biomechanical studies of individual muscle activity.
Methods for crosstalk reduction have been the subject of several investigations.

A number of blind source separation methods was used to reduce the interference. Farina et al. [3] used an instantaneous mixture model, which suppose that there are several electrodes that record the weighted sum of the source signals (the signals from individual muscles). Léouffre et al. [4] showed that the validity of the instantaneous hypothesis is very sensitive to electrode location. For the muscles in which the depolarization propagates along the muscle axis, the instantaneous model is valid only for electrodes lying perpendicularly to muscle direction. When the electrodes are shifted relatively to each other along the muscle axis, the instantaneous model is not valid as the delays appears between the electrodes. Moreover, the muscles must be small to neglect the volume conductor convolutive effects. Jiang and Farina [5] proposed an extension of a BSS technique based on second-order moments (SOBI) for this type of sEMG signal mixtures. The proposed method was based on the transformation of the delayed mixtures to the mixtures of original sources and their first derivatives by the first-order Taylor approximation. The method is applicable only to mixtures with small delay.

Using spatial filtering for multielectrode recordings could be another possibility for crosstalk reduction. Mesin et al. [6] used spatial filtering for crosstalk reduction on simulated signals.

However, there is little published information on using the MUAP propagation to extract real sEMG signals. For real signals, the problem is complicated by several causes. Firstly, muscles consist of a high number of MUs, which are innervated by several motor points. Therefore, MUAP could propagate differently in different MUs of the same muscle. Secondly, it is hard to identify the location of a muscle and its motor points relatively to a surface electrode. And finally, it could be difficult to place a long surface electrode array on a forearm muscle because of a high number of overlappings between muscles. Hence, it could be problematic to perform a $f-k$ filtering for individual muscle signal extraction.

The purpose of this study was to use the direction of MUAP propagation in the extensor digiti minimi (EDM) and 
extensor indicis (EI) muscles to extract the activity of these muscles from real sEMG mixtures by classification of individual MUAPs.

\section{MATERIALS AND METHODS}

\section{A. Model of signal propagation}

EDM and EI muscles were chosen as they are biomechanically independent, i.e. they could be contracted independently of one another, and because of their relative position. These muscles are situated at the dorsal part of a forearm and overlap at the wrist level. EDM lies more proximal than EI muscle, and motor points of EDM muscle are situated more proximal than motor points of EI muscle [1] (Figure 1). The EDC muscle, which also participates in little and index finger extension is situated significantly more proximally than these two muscles and therefore it was not taken into account.

A sEMG signal is a sum of MUAPs, which are observed as peaks. They propagate from motor points (innervation zone) in two opposite directions of muscle endings. As the AP propagation speed is relatively small, each MUAP is detected by two nearby electrodes with a certain delay, which depends on the fiber propagation velocity, propagation direction, and fiber angle. Each MUAP $X_{k}$ was characterized by the delay vector $X_{k}=\left[\begin{array}{ll}\delta_{k}^{12} & \delta_{k}^{23}\end{array}\right]$, where $\delta_{k}^{l m}$ is a delay between the appearance of $X_{k}$ at the $l$-th and the $m$-th electrode.

\section{B. Experimental setting}

sEMG signals $x_{i}$ (where $i=1 \ldots N$ is a channel number, $N=3$ ) were recorded on eight volunteers with no prior known trauma. The linear array of three monopolar electrodes was situated over the intercrossing of the EI and EDM muscles. The inter-electrode distance was $1.5 \mathrm{~cm}$. A ground electrode and a reference electrode were fixed in the wrist, as shown in the Figure 1. The electrodes were placed on the left hand for the first four subjects, and on the right hand for the second four.

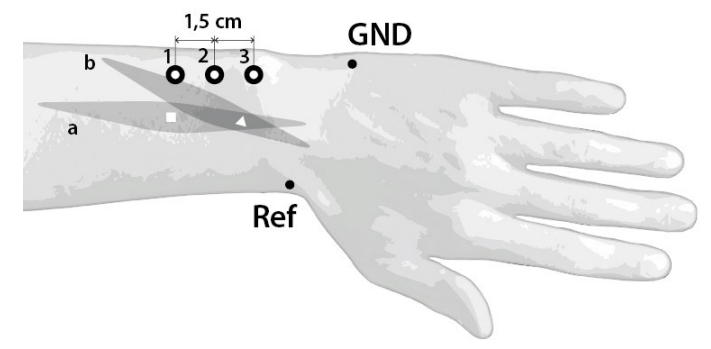

Figure 1. Experimental setup. A square indicates an approximate location of EDM (a) motor points; a triangle indicates an approximate location of EI (b) motor points

An experimental task consisted in performing three finger movements: an extension of a little finger, an extension of an index finger and a simultaneous extension of both little and index fingers. A g.USBamp amplifier was used to record the signal with $2400 \mathrm{~Hz}$ sampling frequency. The signals were filtered backward and forward with order 8 Butterworth filter in $[20 ; 500] \mathrm{Hz}$. The filtered signals are shown in Figure 2. In the same figure the scaled-up 35-ms windows during EDM activity (a) and EI activity (b) are shown.
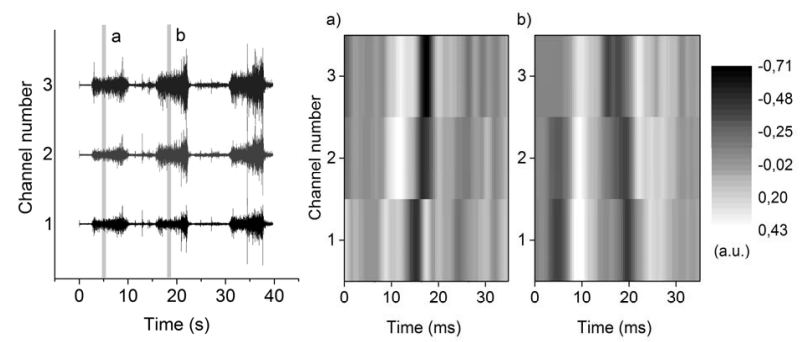

Figure 2. The sEMG recordings $x_{i}$. Scaled-up zones a (during little finger extension) and $\mathrm{b}$ (index finger extension) are shown. The third movement corresponds to simultaneous extension of little and index finger.

The vertical lines in the Figure 2 ( $a$ and $b$ ) correspond to positive (light) and negative (dark) peaks in sEMG signal. The line inclination in the upper and the lower figure is different, which is related to the fact that MUAPs in EDM and EI muscle propagate in different directions.

\section{Peak detection and estimation of propagation direction}

A derivative of sEMGs was used to detect the MUAPs. Only high MUAPs were considered as important; the MUAPs lower than 0.3 of the maximal value of recorded sEMG were considered as noise.

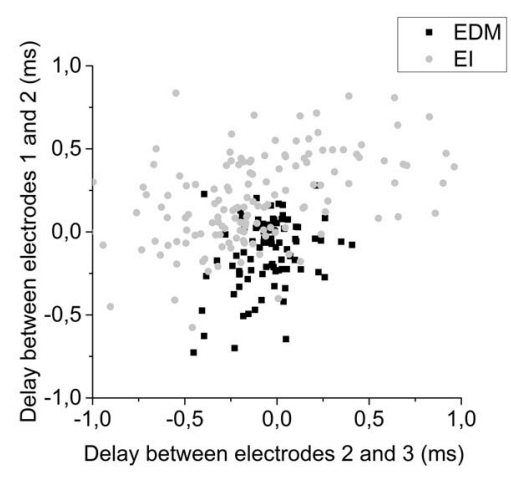

Figure 3. MUAP delay scatter plot

MUAPs were detected in the first channel. A maximum cross-correlation was used to locate a delayed version of each MUAP in the second and the third channels.

\section{Separation}

MUAPs we attributed to one or another muscle according to inter-electrode delays (delay vector).

Two first movements were used to train the classifier, as only one source was active at the same time during each of these movements. When MUAPs were detected, the delay vector was calculated for each MUAP. An example of interelectrode delay scatter plot for EDM and EI activities is shown in the Figure 3. According to these delays, the MUAPs during all three movements were classified into two groups, corresponding to EDM and EI activity by quadratic 
discriminant analysis (QDA). The $k$-th MUAP was attributed to $j$-th muscle activity according to the following rule:

$$
X_{k} \rightarrow\left\{\begin{array}{l}
E D M \quad \text { if } \quad r_{k, e d m}^{2}<r_{k, e i}^{2}+\log \left(\frac{\left|\Sigma_{e i}\right|}{\left|\Sigma_{e d m}\right|}\right) . \\
E I \quad \text { otherwise }
\end{array}\right.
$$

where $r_{k, j}^{2}$ is a Mahalanobis distance between the $k$-th MUAP delay vector and the mean value of the delay vector characterizing the $j$-th muscle activity.

Each movement (EDM and EI) was reconstructed by applying a corresponding mask to the mixture. Each masks permitted to keep the MUAPs attributed to corresponding movement and to hide the other peaks.

\section{RESULTS}

The Figure 4 shows the extracted activity of EDM and EI muscles. It could be seen from the figure, that the peaks are mostly classified as EDM-peaks for the first movement, and as EI-peaks for the second one.

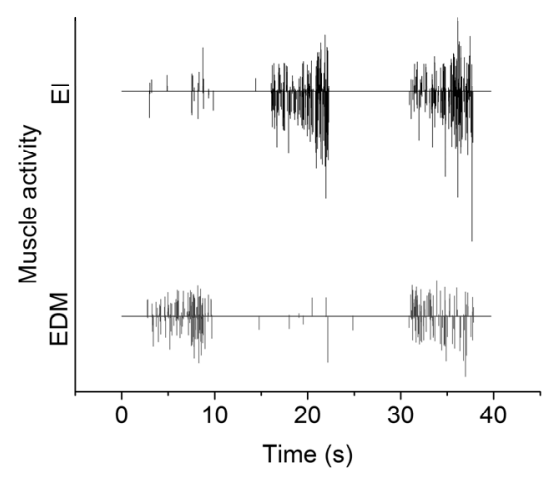

Figure 4. Reconstructed movements

\section{A. The Performance of the Peak Separation Algorithm}

When the signals $s_{j}$ of both muscles $(j=1$ for EI and 2 for EDM) were reconstructed, the signal-to-interference ratio (SIR or also signal-to-crosstalk ratio) and false detected peak fraction (FP) were calculated for both reconstructed signals to estimate the extraction performance.

$S I R_{j}$ was calculated as a ratio between the signal power $P_{j}$ during the movement when the corresponding muscle $j$ was active to the signal power during another movement, when the muscle was not active.

$$
S_{j}(d B)=10 \log _{10}\left(\frac{P_{j}(\text { active })}{P_{j}(\text { inactive })}\right) .
$$

$F P_{j}$ was calculated as a number of MUAPs $N_{j}$ erroneously attributed to $j$-th muscle activity to a total number of MUAPs attributed to this muscle during both movements.

$$
F P_{j}=\frac{N_{j}(\text { inactive })}{N_{j}(\text { inactive })+N_{j}(\text { active })} .
$$

The SIR and FP values were calculated from both extracted signals (Table 1).

TABle 1. SOURCE Extraction PERformanCE

\begin{tabular}{|l|l|l|}
\hline Muscle name & \multicolumn{1}{|c|}{ SIR, dB } & \multicolumn{1}{c|}{ FP } \\
\hline EDM & 12.8 & 0.12 \\
\hline EI & 14.4 & 0.13 \\
\hline Mean & 13.6 & 0.12 \\
\hline
\end{tabular}

\section{B. Comparison of the Peak Separation Algorithm with the Beamformer and the F-JADE}

The proposed algorithm was compared with the beamformer algorithm and the frequency JADE (F-JADE) blind source separation algorithm.

\section{a) Beamformer Algorithm}

The beamformer output was calculated as a weight sum:

$$
s_{j}^{b f}(t)=\frac{1}{N} \sum_{i=1}^{N} w_{i} x_{i}\left(t-[N-i] \cdot \tau_{j}\right),
$$

where $N=3$ is a number of electrodes in a line, $w_{i}$ are weight coefficients. The $w_{i}$ were equal to 1 for each $i$.

\section{b) Blind Source Separation by F-JADE}

The EDM and EI source separation was performed as described in [7]. We considered three recorded sEMG observations $x_{i}$ as convolutive mixtures of EDM and EI signals because of time shift and convolutive effects of the volume conductor. To reformulate the convolutive model into an instantaneous one the observations $x_{i}$ were transferred to timefrequency domain by short-time Fourier transform (STFT). Then two sources were calculated independently in each frequency bin by JADE algorithm. Finally, the source signals were reconstructed by inverse STFT.

The performance of the proposed algorithm was compared with the performance of the beamforming algorithm and FJADE (Table 2).

TABle 2 SIR of The Proposed Method, BEAMFormer AND F-JADE

\begin{tabular}{|l|l|l|l|}
\hline \multirow{2}{*}{ Muscle name } & \multicolumn{3}{|c|}{ SIR, dB } \\
\cline { 2 - 4 } & Peak separation & Beamformer & F-JADE \\
\hline EDM & 12.8 & -0.55 & 3.20 \\
\hline EI & 14.4 & 5.05 & 4.07 \\
\hline Mean & 13.6 & 2.25 & 3.64 \\
\hline
\end{tabular}

The results of the interference reduction by the proposed algorithm, the beamformer, and the F-JADE were also compared with the initial signals $x_{i}$. The gain of each method was calculated as a difference between the SIR-values after interference reduction by this method and the initial SIRvalues. The initial SIR for EDM movement was calculated as a ratio between the power of the first and the second movement in the first channel $(-1.18 \mathrm{~dB})$. The initial SIR for EI movement 
was calculated as a ratio between the power of the second and the first movement in the third channel $(5.59 \mathrm{~dB})$.
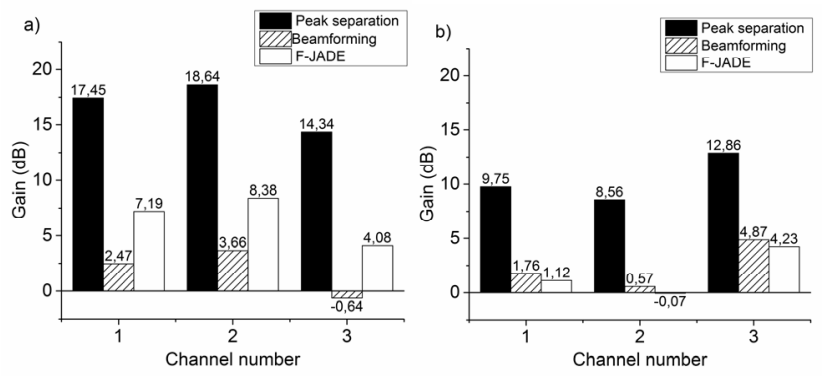

Figure 5. The gain of the proposed method, beamformer and F-JADE calculated for a) EDM movement; b) EI movement.

\section{DISCUSSION}

The performance of the proposed algorithm was shown to be higher than the performance of the beamformer and the FJADE algorithm. Low performance of the beamformer algorithm may be due to differences of MUAPs propagation in different MUs of the same muscle. F-JADE shows the performance higher than the beamformer, but lower than the proposed method. It may be due to the fact that the proposed method performs additional noise reduction by keeping only high MUAPs in extracted signal and neglecting low MUAPs, which may contain noise. However, the loss of information due to neglecting of low MUAPs could be insignificant for biomechanical sEMG signal applications, which exploit the envelope sEMG signals to reconstruct the muscle force [8].

The proposed MUAP separation algorithm was based on QDA, which was used to attribute each MUAP in sEMG mixture to a muscle, either EDM or EI. QDA was used because two groups of MUAPs in 2D delay space could be linearly separated, as it is seen from the Figure 3. The assumption about Gaussian distribution of these groups was also made. To test the normality the Mardia's test was used, which is based on a skewness and a kurtosis of multivariate distribution. For EDM muscle the multivariate skewness of 2D delay distribution was 0.94 and multivariate kurtosis was $9.45(\mathrm{p}=0.0075$ and 0.0438 respectively); for EI muscle the multivariate skewness was 0.30 and multivariate kurtosis was $12.36(\mathrm{p}=0.1269$ and $\left.2.8 \cdot 10^{-11}\right)$. It means that for both groups of MUAPs, from EI and EDM muscle, the normality hypothesis is violated at $95 \%$ significance level. Although normality violation the QDA performance was high.

In addition, it is important to discuss the admissibility of applying the proposed method in case of simultaneous activity of several muscles (the third movement in Figure 2). Sparsity is required to correctly separate the MUAPs. However, this condition may not be satisfied in case of high muscle force level [7]. To study the applicability of the proposed method to these conditions further study should be carried.

\section{CONCLUSION}

The aim of the present research was to extract the EDM and EI muscles sources from the sEMG signals recorded by an electrode array. These muscles were chosen because of their relative position in wrist, which enables to perform separation of individual MUAPs in sEMG signal by time delay.

This study has demonstrated that classification of the individual MUAPs in sEMG mixtures could be more effective than the beamformer or blind separation algorithms. The efficiency of the proposed method could be explained by the fact that it does not require any precise signal propagation model and provides supplementary denoising due to keeping only high peaks in extracted signals. It was shown that it could be difficult to create a precise model of sEMG signal propagation because each muscle consists of a high number of MUs controlled by multiple motor points. Furthermore, from each motor point APs propagate in two different directions.

As one of the main application of sEMG signals is a muscle force estimation, further research is required to reconstruct the muscle force from extracted sources and compare it with the force measured by a sensor. Another possible area of future research would be to apply the proposed method to extract the signals from other muscles, especially from finger flexor muscles, as these signals are important for medical applications, in particular for hand prosthesis control.

\section{ACKNOWLEDGMENT}

The authors thank Anton Andreev (Gipsa-Lab) for his assistance in sEMG signal recording and Dr. Sylvie Charbonnier (Gipsa-Lab) for her valuable comments.

\section{REFERENCES}

[1] E. D. Safwat and E. M. Abdel-Meguid, "Distribution of terminal nerve entry points to the flexor and extensor groups of forearm muscles: An anatomical study," Folia Morphol. (Warsz)., vol. 66, no. 2, pp. 83-93, 2007.

[2] W. Troni, R. Cantello, and I. Rainero, "Conduction velocity along human muscle fibers in situ.," Neurology, vol. 33, no. November, pp. 1453-1459, 1983

[3] D. Farina, C. Févotte, C. Doncarli, and R. Merletti, "Blind Separation of Linear Instantaneous Mixtures of Nonstationary Surface Myoelectric Signals," IEEE Trans. Biomed. Eng., vol. 51, no. 9, pp. 1555-1567, 2004.

[4] M. Léouffre, F. Quaine, and C. Servière, "Testing of instantaneity hypothesis for blind source separation of extensor indicis and extensor digiti minimi surface electromyograms.," J. Electromyogr. Kinesiol., vol. 23, no. 4, pp. 908-15, Aug. 2013.

[5] N. Jiang and D. Farina, "Covariance and time-scale methods for blind separation of delayed sources," IEEE Trans. Biomed. Eng., vol. 58, no. 3 PART 1, pp. 550-556, 2011.

[6] L. Mesin, S. Smith, S. Hugo, S. Viljoen, and T. Hanekom, "Effect of spatial filtering on crosstalk reduction in surface EMG recordings," Med. Eng. Phys., vol. 31, pp. 374-383, 2009.

[7] A. Dogadov, C. Serviere, and F. Quaine, "Blind separation of surface electromyographic mixtures from two finger extensor muscles," Lect. Notes Comput. Sci., vol. 9237, pp. 481-488, 2015.

[8] P. Liu, L. Liu, F. Martel, D. Rancourt, and E. a. Clancy, "Influence of joint angle on EMG-torque model during constant-posture, quasiconstant-torque contractions," J. Electromyogr. Kinesiol., vol. 23, no. 5, pp. 1020-1028, 2013 